\title{
Comunicación Mediada por Ordenador: Preparación de materiales de aprendizaje crítico en torno a los conceptos de identidad y fiabilidad en Internet
}

\section{TERESA LOPEZ-SOTO}

Departamento de Filología

Inglesa (Lengua Inglesa)

Universidad de Sevilla

teresals@us.es

ORCID: https://orcid.org/0000-0001-5583-6321

D.O.I.: http://dx.doi.org/10.12795/JDU.2018.i01.68

Pp.: 1210-1229

\section{Resumen}

Se presenta un resumen de la intervención realizada en un curso de Nuevas Tecnologías aplicadas al estudio lingüístico de la lengua inglesa en el grado de Estudios Ingleses de la Universidad de Sevilla. La propuesta tiene como objetivo incentivar mediante un ciclo de mejora docente (CMD) de 7 horas el desarrollo del espíritu crítico mediante el método investigativo y el trabajo en equipo en un contexto de clase silenciosa por parte del docente. La propuesta se enmarca en el modelo de Aprendizaje Basado en Proyectos (PBL, Project-Based Learning). 
Palabras clave: Nuevas Tecnologías aplicadas al estudio de la Lengua Inglesa; Grado en Estudios Ingleses; docencia universitaria; experimentación docente universitaria; pensamiento crítico.

\section{Breve descripción del contexto de la intervención}

Este ciclo de mejora se ha aplicado a un grupo de estudiantes de la asignatura "Nuevas Tecnologías Aplicadas a la Lengua Inglesa" de 3ㅇ curso del Grado en Estudios Ingleses que ofrece la Universidad de Sevilla. La asignatura se organiza desde el Departamento de Filología Inglesa (Lengua Inglesa) de la Facultad de Filología. El Plan de Estudios se aprobó en Resolución de 20 de diciembre de 2010 de la Universidad de Sevilla (BOE núm.17 de 20 de enero) y en el mismo se recogen 60 créditos optativos. La asignatura incluye restricciones de matriculación pues ha sido, desde su implantación, tradicionalmente muy popular entre los estudiantes. El motivo principal es que en ella encuentran la formación necesaria para abordar el reto que les supone construir un Trabajo Fin de Estudios (Trabajo Fin de Grado, TFG) con las suficientes garantías. En el curso académico 2018-19 se ha restringido el número de posibles matriculados a 35. Este ciclo de mejora se ha aplicado al Grupo 1 de la asignatura, que cuenta con un total de 39 estudiantes (4 de ellos han sido admitidos a petición propia y mediante autorización de la coordinadora).

La docencia de la asignatura se imparte en un aula inteligente, que cuenta con 50 puestos informáticos, sistema integrado de control docente por ordenador, puesto informático específico para el docente, pizarra digital, pizarra blanca y cuenta con una calidad óptima de luminosidad y sistema de calefacción y aire acondicionado. Todos los estudiantes cuentan con un puesto informático perfectamente integrado, con micrófono y auriculares conectados, en línea con el puesto docente centralizado y con acceso a todos los recursos informáticos necesarios para seguir la 
docencia. Los puestos informáticos de los alumnos se integran en filas.

\section{Diseño previo del ciclo de mejora docente}

Este CMD se denomina "Comunicación mediada por ordenador: preparación de materiales de aprendizaje crítico en torno a los conceptos de identidad y fiabilidad en Internet". Antes de pasar a describir la metodología posible diseñada para este CMD es preciso indicar los objetivos que se buscaban con el mismo. La asignatura se estructura en una serie de tareas de aprendizaje eminentemente individual con las que el estudiante puede incrementar la complejidad del estudio de la lengua. Se trata, fundamentalmente, de asignaturas de acceso a información, de manejo, creación y procesamiento de bases de datos lingüísticas, de programas de cribado de la información y otras herramientas de naturaleza estadística o del campo de la lingüística aplicada (traducción asistida por ordenador, bases de datos terminológicas, entre otras). Como parte del proceso, e importante para la evaluación diseñada, el estudiante debe redactar un artículo de revisión crítica y otro de naturaleza empírica a partir de la observación de datos. Puesto que gran parte de la docencia se ha enfocado tradicionalmente al aprendizaje de herramientas informáticas, la calidad de los análisis y razonamientos críticos que debían hacer los estudiantes como culminación del proceso de aprendizaje en los dos artículos científicos a redactar se veía seriamente afectada. En resumen, el estudiante adquiría destrezas complejas pero no desarrollaba las habilidades necesarias que las mismas requieren: toma de decisiones, análisis crítico.

Este CMD pretende dar respuesta a esa limitación mediante la incorporación de una nueva metodología de naturaleza constructivista, que se inspira además en el aprendizaje por problemas y en el planteamiento de 
preguntas enigma, preguntas motivadoras para provocar la reacción del estudiante en la consecución de las actividades programadas. Además, el modelo metodológico posible sigue la dinámica del aprendizaje colaborativo incentivando el trabajo en equipo y alejándose del trabajo individual que venía haciéndose en el aula.

\section{Mapa de Contenidos}

El mapa de contenidos de la CMD se puede observar en la Figura 1. La gráfica utiliza recuadros punteados para ampliar zonas de contenidos que giran en torno a unas actividades concretas. Otro elemento gráfico que se usa son las flechas/líneas. Cuando son de doble dirección indican que se da la misma importancia a un contenido que a otro. Cuando son líneas rectas, indican correlación entre las partes. Las flechas unidireccionales se utilizan únicamente para referenciar contenidos jerarquizados, es decir, cuando un contenido concreto se desgrana en otro de nivel inferior.

Jornadas de Formación e Innovación Docente del Profesorado | № 1 (2018) Esta obra se distribuye con la licencia Creative Commons 


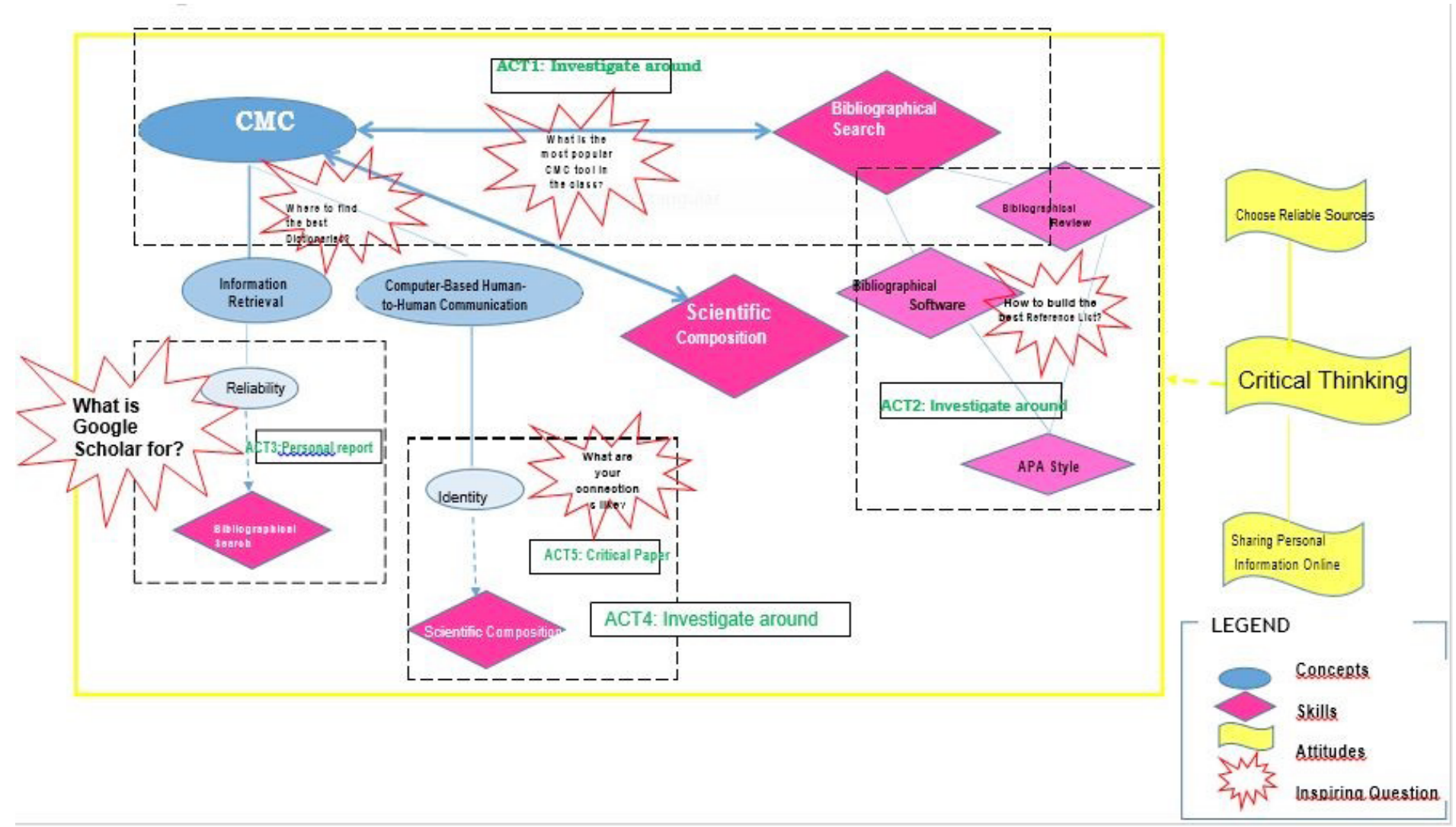

Figura 1. Mapa de contenidos conceptuales, procedimentales y actitudinales del CMD

Jornadas de Formación e Innovación Docente del Profesorado | № 1 (2018)

(c) E $\Theta$ Esta obra se distribuye con la licencia Creative Commons

(c) $(7) \Theta$ RY Reconocimiento-NoComercial-SinObraDerivada

Internacional (CC BY-NC-ND 4.0.) 
La distribución por colores y formas es la siguiente:

- Azul para contenidos conceptuales, morado para contenidos procedimentales, amarillo para contenidos actitudinales.

- Forma elíptica para contenidos conceptuales, romboide para contenidos procedimentales, forma de bandera para contenidos actitudinales.

- Estrellas para preguntas enigma y recuadros en verde para actividades programadas.

El esquema gráfico debe entenderse como que los contenidos correlacionados en la parte superior se desarrollan primero y se siguen por otros de nivel inferior que se desarrollan a continuación. Los contenidos actitudinales estarán presentes a lo largo de todo el CMD.

A continuación se detallan los aspectos importantes de dicho mapa de contenido:

1. Contenidos conceptuales. Los contenidos conceptuales más importantes son los de "identidad" y "fiabilidad". Por "identidad" entendemos aspectos que tienen que ver con nuestra imagen online: anonimidad, características personales que damos a conocer (self-disclosure) y toda una serie de aspectos que tienen que ver con la identidad personal, la social, la colectiva, entre otras. Por "fiabilidad" en la red entendemos aquellos aspectos de confianza y pedigree en las fuentes que consultamos que nos ayudan a caracterizar los contenidos entre ciertos, probables o inseguros.

2. Contenidos procedimentales. Se trabajan los siguientes: herramientas de acceso a información bibliográfica, herramientas de manejo de referencias bibliográficas, estilo de redacción APA y técnicas de redacción de artículos científicos. 
3. Contenidos actitudinales. Este CMD se centra en ayudar al estudiante a utilizar de manera crítica las herramientas, y no a utilizarlas como un fin en sí mismas. Este espíritu crítico debe, además, desarrollarse en dos aspectos importantes y que les serán de utilidad en su práctica profesional futura: el manejo correcto de su imagen personal en redes sociales y la toma de decisiones que les permitan elegir los mejores recursos online para fines profesionales o académicos.

\section{Modelo Metodológico Posible}

Este modelo metodológico posible parte de la hipótesis que el espíritu crítico puede desarrollarse solo cuando existe un auténtico intercambio de ideas. Siguiendo a King y Kitchener (1994), existen hasta 7 etapas para desarrollar el juicio reflexivo, y entre ellas está la recepción de nuevos conceptos y la reelaboración de los antiguos. Por otra parte, el aprendizaje basado en problemas (Hung et al, 2008) establece que cuando el estudiante debe resolver por sí mismo un reto que se le plantea, es cuando realmente desarrolla los contenidos implicados en el proceso de enseñanza-aprendizaje. Esto enlaza además con la teoría de Finkel (2008), partidario de la clase silenciosa, que es aquella en la que el docente prácticamente no participa en la creación de contenido, sino que observa y ayuda al estudiante a crearlo. Finkel además desarrolló un modelo denominado de "talleres conceptuales", altamente sofisticado los cuales se definen como lugares de encuentro en el aula donde todo el proceso de aprendizaje se sustenta en el razonamiento. El estudiante recibe una pregunta enigma escrita en un papel que deberá solucionar, razonar o definir en grupos de 4 personas. La exposición de la pregunta se hace por escrito para despersonalizar la figura del docente durante todo el proceso. Además, al estar escrito, permanece durante todo el tiempo en el que se 
está realizando la actividad. Este modelo se conecta también con el concepto de "aprendizaje crítico natural". Bain (2006) estableció que el "aprendizaje crítico natural" es crítico por naturaleza, y se basa en la reformulación constante de lo aprendido, a partir de ejemplos y mediante la aplicación del método prueba-error. En primer lugar, el docente, según este autor, desafía a los estudiantes a resolver los problemas, animándolos a buscar evidencias que sustenten sus propios razonamientos. Esta confrontación del concepto con la realidad facilitaría el aprendizaje a largo plazo.

Este tipo de enfoque basado en la resolución de problemas, es bastante similar al modelo conocido como Aprendizaje Basado en Proyectos (Project-Based Learning, PBL de sus siglas en inglés). Existen experiencias recogidas en asignaturas similares, donde los estudiantes también se organizan en equipos para conseguir dinamizar cursos del área de las TIC. Ardaiz-Villanueva et al (2011) describen una experiencia similar al contexto que aquí se presenta en donde concluyen que los equipos que tuvieron que enfrentarse a la resolución de proyectos y que estaban formado por estudiantes con similares niveles de afinidad y creatividad, consiguieron mejores resultados. Por último, es importante resaltar que todo el modelo aquí expuesto no es solo construccionista, sino también incentiva el aprendizaje colaborativo y cooperativo. Si aceptamos que el aprendizaje cooperativo es aquel en el que el docente todavía tiene parte de responsabilidad en la elaboración de los contenidos en el aula, al trabajar conjuntamente pero activamente con el estudiante, este modelo metodológico posible hace hincapié en el aprendizaje colaborativo puesto que se pretende, siguiendo a Finkel (2008), que la docente no participe en absoluto en la propuesta de actividades. En cualquier caso, el modelo metodológico posible debe entenderse como uno en el que se tienen en cuenta los siguientes aspectos: 
a) Se trabaja en equipo.

b) Se plantea una pregunta motivadora, a modo de problema a resolver.

c) Se distingue entre experto y usuario.

d) Implica investigar para encontrar las respuestas a partir de consultas a expertos y usuarios.

\section{Secuencia de Actividades Programadas}

Todas las actividades están diseñadas para ser realizadas en grupos de 5 personas. Los grupos se formarán de manera espontánea en el aula, sin indicaciones de la docente. Las instrucciones de funcionamiento de los equipos incluyen dos aspectos importantes. En primer lugar, todos los equipos deben repartir roles entre todos sus miembros. Serán los estudiantes quienes decidan qué roles serán estos y cómo se reparten. En segundo lugar, deberán acordar una serie de reglas de funcionamiento interno dentro del equipo en relación a los objetivos que se marquen en las diferentes actividades. Tanto la división por roles como las reglas de funcionamiento interno se enviarán por la plataforma virtual a la docente.

Las actividades siguen, todas ellas, a una o dos preguntas inspiradoras (preguntas enigma siguiendo la terminología de Finkel (2008)). Se puede considerar que las mismas inician un proyecto o reto que cada equipo debe conseguir por separado. El método indicado por la docente para la terminación del proyecto será el de investigar y crear. La fase de investigación que deberá llevar a cabo cada equipo consistirá en la consulta a fuentes expertas y usuarias. Los conceptos "experto" y "usuario" en este contexto se explican al comienzo de las actividades. Se entiende por "experto" una fuente ya validada; por ejemplo: fuentes académicas con reconocida fiabilidad o especialistas en la materia. Se considerarán "usuarios" cualquier otra persona que hace uso o participa de dicho fenómeno 
sin que pueda ser considerada estrictamente como especialista en la materia. A continuación, y una vez investigado y analizado el fenómeno planteado (pregunta inspiradora), los estudiantes tendrán que crear: un informe por escrito, una presentación oral final y un debate con el resto de la clase. Con este esquema de trabajo, por tanto, se consigue un doble intercambio de información que puede favorecer el desarrollo del pensamiento crítico:

a) Recogida de información directamente de partes externos (expertos o usuarios).

b) Valoración de los resultados obtenidos en debate interno y de aula.

Tabla 1. Actividades programadas

\begin{tabular}{|l|l|l|l|l|}
\hline Sesión & Actividad & Duración & Finalidad & Contenidos/Objetivos \\
\hline 0 & $\begin{array}{l}\text { CUESTIONARIO } \\
\text { INICIAL }\end{array}$ & Indefinida & $\begin{array}{l}\text { Conocer el plan- } \\
\text { teamiento } \\
\text { mental del estu- } \\
\text { diante referente } \\
\text { al fenómeno a } \\
\text { estudiar }\end{array}$ & $\begin{array}{l}\text { Recopilación de con- } \\
\text { tenidos relativos al } \\
\text { CMD }\end{array}$ \\
\hline 1 & $\begin{array}{l}\text { Investiga cuál } \\
\text { es la herra- } \\
\text { mienta de CMC } \\
\text { más popular } \\
\text { en el aula }\end{array}$ & $\begin{array}{l}\text { Conocer y con- } \\
\text { trastar las opi- } \\
\text { niones propias } \\
\text { con las del resto } \\
\text { de la clase. De- } \\
\text { sarrollar habili- } \\
\text { dades de trabajo } \\
\text { en equipo }\end{array}$ & $\begin{array}{l}\text { Herramientas de co- } \\
\text { municación me- } \\
\text { diada por ordenador. } \\
\text { Caracteristicas y } \\
\text { funcionalidades. }\end{array}$ \\
\hline $\begin{array}{l}\text { Investiga cuál } \\
\text { es el diccio- } \\
\text { nario online } \\
\text { major refe- } \\
\text { renciado se- } \\
\text { gún tus } \\
\text { compañeros }\end{array}$ & $30^{\prime}$ & $\begin{array}{l}\text { Desarrollar el } \\
\text { espíritu crí- } \\
\text { tico en la toma } \\
\text { de decisiones } \\
\text { profesionales }\end{array}$ & $\begin{array}{l}\text { Fiabilidad en los re- } \\
\text { cursos obtenidos a } \\
\text { través de Internet }\end{array}$ \\
\hline
\end{tabular}

Jornadas de Formación e Innovación Docente del Profesorado | № 1 (2018) Esta obra se distribuye con la licencia Creative Commons Reconocimiento-NoComercial-SinObraDerivada Internacional (CC BY-NC-ND 4.0.) 


\begin{tabular}{|c|c|c|c|c|}
\hline & $\begin{array}{l}\text { Presentación y } \\
\text { debate final }\end{array}$ & $30^{\prime}$ & $\begin{array}{l}\text { Practicar habi- } \\
\text { lidades comu- } \\
\text { nicativas orales } \\
\text { y contraste de } \\
\text { opiniones } \\
\text { conjuntas. }\end{array}$ & Foros de discusión \\
\hline \multirow[t]{3}{*}{2} & $\begin{array}{l}\text { Investiga cómo } \\
\text { crear una lista } \\
\text { de referencias } \\
\text { lo suficiente- } \\
\text { mente fiable } \\
\text { para incluirla } \\
\text { como modelo } \\
\text { en tu actividad } \\
\text { académica }\end{array}$ & $30^{\prime}$ & $\begin{array}{l}\text { Conocer y con- } \\
\text { trastar las opi- } \\
\text { niones propias } \\
\text { con las del resto } \\
\text { de la clase. De- } \\
\text { sarrollar habili- } \\
\text { dades de trabajo } \\
\text { en equipo }\end{array}$ & $\begin{array}{l}\text { Herramientas de re- } \\
\text { cuperación de infor- } \\
\text { mación bibliográfica } \\
\text { online. Software de al- } \\
\text { macenamiento biblio- } \\
\text { gráfico. Normas de } \\
\text { estilo APA }\end{array}$ \\
\hline & $\begin{array}{l}\text { Describe bre- } \\
\text { vemente la } \\
\text { herramienta } \\
\text { Google Scholar } \\
\text { y comparte en } \\
\text { tu equipo }\end{array}$ & $30^{\prime}$ & $\begin{array}{l}\text { Conocer y con- } \\
\text { trastar las opi- } \\
\text { niones propias } \\
\text { con las del resto } \\
\text { de la clase. De- } \\
\text { sarrollar habili- } \\
\text { dades de trabajo } \\
\text { en equipo }\end{array}$ & $\begin{array}{l}\text { Herramientas de re- } \\
\text { cuperación de infor- } \\
\text { mación bibliográfica } \\
\text { online }\end{array}$ \\
\hline & $\begin{array}{l}\text { Presentación y } \\
\text { debate final }\end{array}$ & $30^{\prime}$ & $\begin{array}{l}\text { Practicar habi- } \\
\text { lidades comu- } \\
\text { nicativas orales } \\
\text { y contraste de } \\
\text { opiniones } \\
\text { conjuntas. }\end{array}$ & Foros de discusión \\
\hline 3 & $\begin{array}{l}\text { Investiga los } \\
\text { perfiles en red } \\
\text { de tus com- } \\
\text { pañeros de } \\
\text { equipo. Com- } \\
\text { parte la visión } \\
\text { que tienes de } \\
\text { ti mismo en } \\
\text { diferentes re- } \\
\text { des sociales } \\
\text { con las opi- } \\
\text { niones de tu } \\
\text { equipo }\end{array}$ & $60^{\prime}$ & $\begin{array}{l}\text { Conocer y con- } \\
\text { trastar las opi- } \\
\text { niones propias } \\
\text { con las del resto } \\
\text { de la clase. De- } \\
\text { sarrollar habili- } \\
\text { dades de trabajo } \\
\text { en equipo }\end{array}$ & $\begin{array}{l}\text { Identidad en herra- } \\
\text { mientas de comuni- } \\
\text { cación mediada por } \\
\text { ordenador }\end{array}$ \\
\hline
\end{tabular}




\begin{tabular}{|c|c|c|c|c|}
\hline & $\begin{array}{l}\text { Presentación y } \\
\text { debate final }\end{array}$ & $30^{\prime}$ & $\begin{array}{l}\text { Practicar habi- } \\
\text { lidades comu- } \\
\text { nicativas orales } \\
\text { y contraste de } \\
\text { opiniones } \\
\text { conjuntas. }\end{array}$ & Foros de discusión \\
\hline \multirow[t]{2}{*}{4} & $\begin{array}{l}\text { Revisa todos } \\
\text { los borrado- } \\
\text { res de artí- } \\
\text { culo científico } \\
\text { crítico que } \\
\text { han escrito } \\
\text { tus compañe- } \\
\text { ros de equipo } \\
\text { y elige el me- } \\
\text { jor(es) indi- } \\
\text { cando el por } \\
\text { qué. }\end{array}$ & $60^{\prime}$ & $\begin{array}{l}\text { Desarrollar es- } \\
\text { píritu autocrí- } \\
\text { tico y valorar el } \\
\text { trabajo del otro. } \\
\text { Aprender a eva- } \\
\text { luar contenidos } \\
\text { propios y ajenos } \\
\text { utilizando he- } \\
\text { rramientas di- } \\
\text { señadas por } \\
\text { consenso }\end{array}$ & $\begin{array}{l}\text { Diseño de un artículo } \\
\text { científico crítico }\end{array}$ \\
\hline & $\begin{array}{l}\text { Presentación y } \\
\text { debate final }\end{array}$ & $30^{\prime}$ & $\begin{array}{l}\text { Practicar habi- } \\
\text { lidades comu- } \\
\text { nicativas orales } \\
\text { y contraste de } \\
\text { opiniones } \\
\text { conjuntas. }\end{array}$ & Foros de discusión \\
\hline 4 & $\begin{array}{l}\text { CUESTIONARIO } \\
\text { FINAL }\end{array}$ & $\begin{array}{l}\text { Indefi- } \\
\text { nido }\end{array}$ & & \\
\hline
\end{tabular}

\section{Cuestionario inicial-final}

Al inicio del ciclo, y previo a la primera sesión, los estudiantes contestaron un cuestionario inicial de 10 preguntas en torno a los siguientes conceptos:

a) Definición del campo de la Comunicación mediada por Ordenador.

b) Aspectos de fiabilidad en la recuperación de información online.

c) Aspectos de identidad en la Comunicación mediada por Ordenador.

d) Descripción de herramientas de almacenamiento bibliográfico. 


\section{Aplicación del CMD}

\section{Relato resumido de las sesiones}

Durante la primera sesión se les explica a los estudiantes que durante las siguientes sesiones se va a terminar el módulo del curso denominado "Computer-Mediated Communication". En la plataforma virtual, y previo a este ciclo de mejora, se publica el cuestionario inicial, pero en ningún momento se les dice a los estudiantes el objetivo del mismo (26 estudiantes lo completan). Centrándome en la primera sesión del ciclo de mejora, como decía, informo a los estudiantes de que la actividad de las siguientes sesiones ( 4 en total) será en grupo. Esto no es recibido por agrado, la mayor parte de la clase lo acepta indiferente, y un grupo importante se opone. Es complicado dinamizar el aula. Los estudiantes están en mitad de una tarea muy importante para ellos, la redacción de un artículo crítico, y no parece conformes a realizar actividades de este tipo. Estoy convencida de que consideran que el trabajo en equipo es una pérdida de tiempo.

En general, la impresión que recibo es que quieren terminar con la tarea, no se involucran. Esta valoración se me hace evidente cuando al final de la clase, en la presentación final, emiten brevemente los resultados de su trabajo en las dos actividades. El trabajo de actividades se hace en base a la investigación. Les explico que pueden contactar con expertos (la profesora es experta, las fuentes bibliográficas son expertas) y usuarios (los usuarios son los mismos estudiantes y cualquier otra persona no experta). Les indico que pueden salir del aula, sentarse como quieran. Pero no lo hacen, se quedan exactamente en su sitio, que es el lugar que normalmente ocupan en todas las sesiones. El objetivo de todo este ciclo de mejora era incrementar su capacidad crítica. No obstante, parece que no se 
logra, porque en la presentación final no aportan ningún tipo de información que no sea estrictamente objetiva. Se limitan a preguntar en el aula y a recopilar las respuestas obtenidas.

La actividad del segundo día continúa con la misma estructura: trabajo en grupos, tareas asignadas por los propios estudiantes y metodología basada en la investigación. Para el día 2 los alumnos habían tenido que enviar un informe de su producción del día anterior, en el que realizaron 2 actividades. Analizando sus informes, puedo observar que siguen limitándose a enunciar los datos. Puesto que la actividad de equipo consiste en explorar alrededor para buscar las respuestas a las preguntas, en el informe del día 1 (actividades 1 y 2) los equipos siguen limitándose exclusivamente a enunciar las respuestas que han obtenido. Las mismas se limitan a preguntarme a mí directamente (me consideran experta) y a sus compañeros en el aula. El hecho de que las primeras actividades incluyeran preguntas del tipo "cuántas personas en el aula" los orientan a ceñirse exclusivamente a ese campo. Es decir, las actividades, lejos de fomentar la autonomía y en especial el pensamiento crítico, siguen realizándose en un entorno constreñido por las instrucciones básicas que reciben. El ambiente está mucho más relajado.

La tercera sesión comienza diferente porque los estudiantes han trabajado fuera del aula. Sin embargo, no funciona porque al limitarse los equipos a anotar los datos, se convierte en una secuencia de oraciones que no propicia el debate. Llegados a este punto se modifica la actividad propuesta y se cambia por una mucho más ambiciosa y que los obliga a moverse. En el equipo, deben revisar todos los borradores del artículo crítico que deben entregar la semana siguiente y elegir cuál es el mejor. Para ello, 
deben seguir con la dinámica de trabajo en equipo e investigar alrededor. En esta ocasión, sí que se involucran y se produce un interesante debate final que no estaba previsto. Los equipos intervienen (sólo los portavoces lo hacen porque se insistió que las reglas de trabajo de los equipos debían respetarse) y se van anotando las ideas en la pizarra.

La última sesión comienza diferente porque los estudiantes llevan varios días trabajando en la nueva dinámica. En esta ocasión, sí que se involucran y se produce un interesante debate final. Los equipos intervienen y se van anotando las ideas en la pizarra. Los estudiantes están mucho más relajados en la última sesión del segundo ciclo de mejora. La actividad de búsqueda del mejor artículo en el equipo ha sido la que más les ha motivado. Todos los grupos, menos uno, han expuesto en público las características que los mejores artículos de sus respectivos equipos tienen desde su perspectiva. Los estudiantes además han creado buenos lazos, pues la solidaridad, el respeto se han apreciado en todas las tareas, pero especialmente en esta última.

\section{Evaluación del aprendizaje de los estudiantes}

Para evaluar el aprendizaje de los estudiantes durante el ciclo se ha diseñado un cuestionario que aparece descrito en una sección anterior. En la Tabla 2 se muestra la rúbrica correspondiente a cada nivel. En la Figura 2 se muestran los resultados divididos por niveles, de la A a la E para el cuestionario inicial. La Figura 3 muestra los resultados de este cuestionario final. 
Tabla 2. Rúbrica del cuestionario inicial-final

\begin{tabular}{|c|c|c|c|c|c|}
\hline & A & B & C & D & $E$ \\
\hline 1 & $\begin{array}{l}\text { Define el con- } \\
\text { cepto y los } \\
\text { aspectos de fia- } \\
\text { bilidad, identi- } \\
\text { dad y ejemplos }\end{array}$ & $\begin{array}{l}\text { Define el con- } \\
\text { cepto y los as- } \\
\text { pectos de } \\
\text { fiabilidad, } \\
\text { identidad }\end{array}$ & $\begin{array}{l}\text { Define el } \\
\text { concepto }\end{array}$ & $\begin{array}{l}\text { Hace una valo- } \\
\text { ración coloquial } \\
\text { a la pregunta }\end{array}$ & $\begin{array}{l}\text { No contesta/ } \\
\text { Contesta } \\
\text { erróneamente }\end{array}$ \\
\hline 2 & $\begin{array}{l}\text { Define de ma- } \\
\text { nera crítica a la } \\
\text { pregunta }\end{array}$ & $\begin{array}{l}\text { Define de ma- } \\
\text { nera objetiva } \\
\text { con ejemplos }\end{array}$ & $\begin{array}{l}\text { Define de } \\
\text { manera } \\
\text { objetiva }\end{array}$ & $\begin{array}{l}\text { Hace una valo- } \\
\text { ración personal } \\
\text { de la pregunta }\end{array}$ & $\begin{array}{l}\text { No contesta/ } \\
\text { Contesta } \\
\text { erróneamente }\end{array}$ \\
\hline 3 & $\begin{array}{l}\text { Afirma posi- } \\
\text { tivamente y } \\
\text { detalla las con- } \\
\text { notaciones del } \\
\text { fenómeno }\end{array}$ & $\begin{array}{l}\text { Afirma positi- } \\
\text { vamente y des- } \\
\text { cribe de manera } \\
\text { genérica el } \\
\text { fenómeno }\end{array}$ & $\begin{array}{l}\text { Afirma po- } \\
\text { sitivamente } \\
\text { sin des- } \\
\text { cribir el } \\
\text { fenómeno }\end{array}$ & $\begin{array}{l}\text { Afirma de ma- } \\
\text { nera negativa } \\
\text { con justificación }\end{array}$ & $\begin{array}{l}\text { Afirma de } \\
\text { manera ne- } \\
\text { gativa sin jus- } \\
\text { tificación /No } \\
\text { contesta }\end{array}$ \\
\hline 4 & $\begin{array}{l}\text { Afirma posi- } \\
\text { tivamente y } \\
\text { detalla las con- } \\
\text { notaciones del } \\
\text { fenómeno }\end{array}$ & $\begin{array}{l}\text { Afirma positi- } \\
\text { vamente y des- } \\
\text { cribe de manera } \\
\text { genérica el } \\
\text { fenómeno }\end{array}$ & $\begin{array}{l}\text { Afirma po- } \\
\text { sitivamente } \\
\text { sin des- } \\
\text { cribir el } \\
\text { fenómeno }\end{array}$ & $\begin{array}{l}\text { Afirma de ma- } \\
\text { nera negativa } \\
\text { con justificación }\end{array}$ & $\begin{array}{l}\text { No contesta/ } \\
\text { Contesta } \\
\text { erróneamente }\end{array}$ \\
\hline 5 & $\begin{array}{l}\text { Afirma posi- } \\
\text { tivamente y } \\
\text { detalla las con- } \\
\text { notaciones del } \\
\text { fenómeno }\end{array}$ & $\begin{array}{l}\text { Afirma positi- } \\
\text { vamente y des- } \\
\text { cribe de manera } \\
\text { genérica el } \\
\text { fenómeno }\end{array}$ & $\begin{array}{l}\text { Afirma po- } \\
\text { sitivamente } \\
\text { sin des- } \\
\text { cribir el } \\
\text { fenómeno }\end{array}$ & $\begin{array}{l}\text { Afirma de ma- } \\
\text { nera negativa } \\
\text { con justificación }\end{array}$ & $\begin{array}{l}\text { No contesta/ } \\
\text { Contesta } \\
\text { erróneamente }\end{array}$ \\
\hline 6 & $\begin{array}{l}\text { Incorpora no- } \\
\text { tables aporta- } \\
\text { ciones críticas } \\
\text { mencionando un } \\
\text { modelo teórico }\end{array}$ & $\begin{array}{l}\text { Incorpora nota- } \\
\text { bles aportacio- } \\
\text { nes críticas sin } \\
\text { mencionar nin- } \\
\text { gún modelo }\end{array}$ & $\begin{array}{l}\text { Describe el } \\
\text { modelo sin } \\
\text { aportacio- } \\
\text { nes críticas }\end{array}$ & $\begin{array}{l}\text { Hace una mera } \\
\text { valoración } \\
\text { coloquial }\end{array}$ & $\begin{array}{l}\text { No contesta/ } \\
\text { Contesta } \\
\text { erróneamente }\end{array}$ \\
\hline 7 & $\begin{array}{l}\text { Incorpora no- } \\
\text { tables aporta- } \\
\text { ciones críticas } \\
\text { mencionando un } \\
\text { modelo teórico }\end{array}$ & $\begin{array}{l}\text { Incorpora nota- } \\
\text { bles aportacio- } \\
\text { nes críticas sin } \\
\text { mencionar nin- } \\
\text { gún modelo }\end{array}$ & $\begin{array}{l}\text { Describe el } \\
\text { modelo sin } \\
\text { aportacio- } \\
\text { nes críticas }\end{array}$ & $\begin{array}{l}\text { Hace una mera } \\
\text { valoración } \\
\text { coloquial }\end{array}$ & $\begin{array}{l}\text { No contesta/ } \\
\text { Contesta } \\
\text { erróneamente }\end{array}$ \\
\hline 8 & $\begin{array}{l}\text { Define el con- } \\
\text { cepto con } \\
\text { ejemplos }\end{array}$ & $\begin{array}{l}\text { Define el con- } \\
\text { cepto sin } \\
\text { ejemplos }\end{array}$ & $\begin{array}{l}\text { Solo aporta } \\
\text { ejemplos }\end{array}$ & $\begin{array}{l}\text { Hace una valo- } \\
\text { ración personal }\end{array}$ & $\begin{array}{l}\text { No contesta/ } \\
\text { Contesta } \\
\text { erróneamente }\end{array}$ \\
\hline
\end{tabular}

Jornadas de Formación e Innovación Docente del Profesorado | № 1 (2018) Esta obra se distribuye con la licencia Creative Commons 


\begin{tabular}{|c|l|l|l|l|l|}
\hline 9 & $\begin{array}{l}\text { Describe el con- } \\
\text { cepto con to- } \\
\text { das sus } \\
\text { connotaciones }\end{array}$ & $\begin{array}{l}\text { Define el con- } \\
\text { cepto de ma- } \\
\text { nera genérica }\end{array}$ & $\begin{array}{l}\text { Solo hace } \\
\text { referen- } \\
\text { cia al con- } \\
\text { cepto de } \\
\text { copyright }\end{array}$ & $\begin{array}{l}\text { Solo hace refe- } \\
\text { rencia al diseño } \\
\text { de página web }\end{array}$ & $\begin{array}{l}\text { No contestal } \\
\text { Contesta } \\
\text { erróneamente }\end{array}$ \\
\hline 10 & $\begin{array}{l}\text { Describe todos } \\
\text { los apartados } \\
\text { con explicacio- } \\
\text { nes detalladas }\end{array}$ & $\begin{array}{l}\text { Describe to- } \\
\text { dos los aparta- } \\
\text { dos de manera } \\
\text { genérica }\end{array}$ & $\begin{array}{l}\text { Describe } \\
\text { los apar- } \\
\text { tados más } \\
\text { importantes }\end{array}$ & $\begin{array}{l}\text { Describe po- } \\
\text { cos apartados } \\
\text { yo los más } \\
\text { relevantes }\end{array}$ & $\begin{array}{l}\text { No contesta/ } \\
\text { Contesta } \\
\text { erróneamente }\end{array}$ \\
\hline
\end{tabular}

De las figuras siguientes se deduce que no hubo gran diferencia entre los resultados obtenidos del cuestionario inicial y del final. Al tratarse de un ciclo de docencia breve, parte de los contenidos habían sido obviamente ya presentados en clase. No obstante, cabe resaltar el comportamiento de los estudiantes en relación a las preguntas 5 y 7 del cuestionario:

5.Is "information retrieval" online similar in its parameters to communication online? How similar? How different?

7.Are you aware of your own presence (profile picture, personal data and posts) when you use social networks? How?

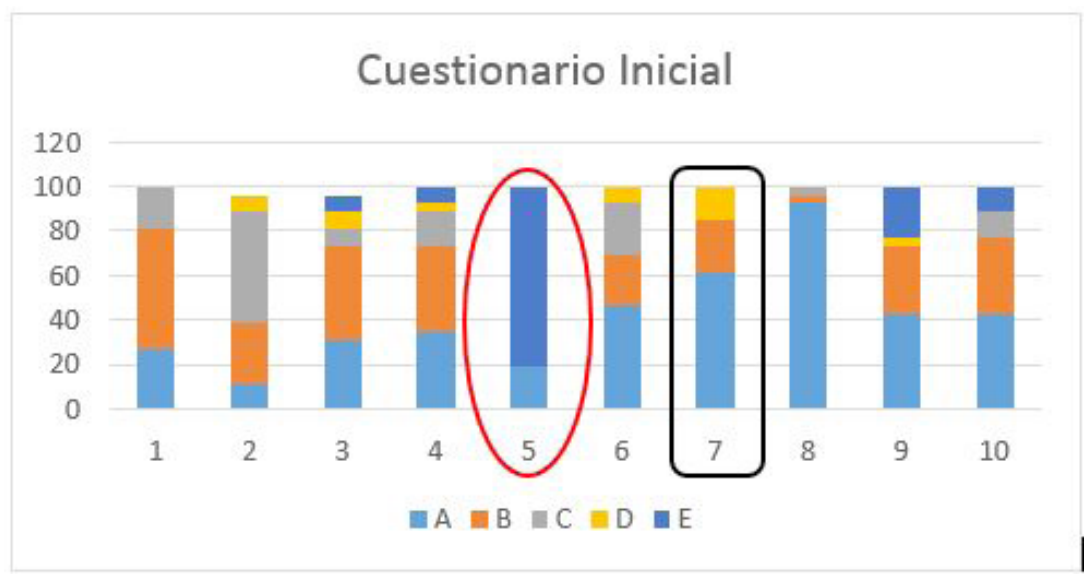

Figura 2. Resultados del cuestionario inicial

Jornadas de Formación e Innovación Docente del Profesorado | № 1 (2018) Esta obra se distribuye con la licencia Creative Commons 


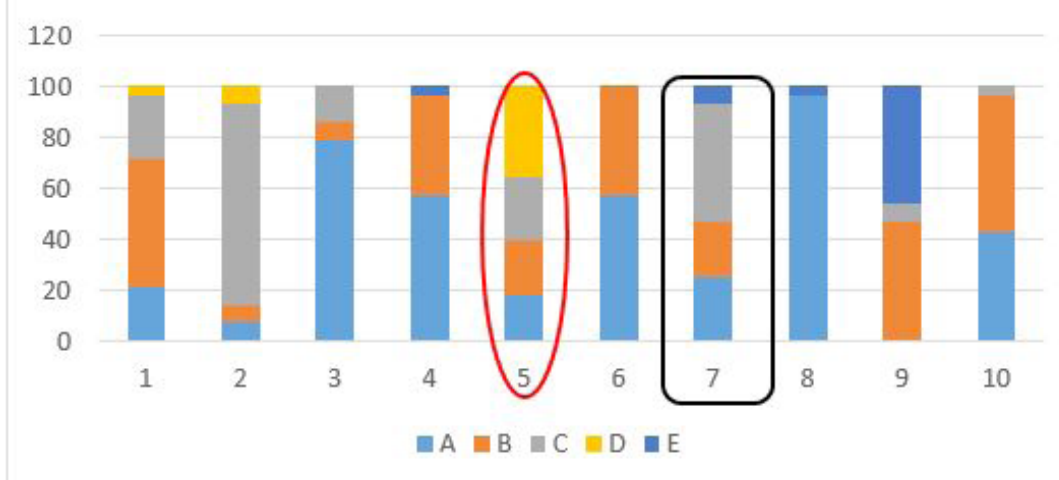

Figura 3. Resultados del cuestionario final

Las dos preguntas se refieren a conceptos más críticos dentro del cuestionario. Las mismas, hacen referencia a relaciones que no se han estudiado en el curso, en primer lugar, si existe una similitud entre recuperación de información y comunicación en cuanto a los parámetros que las definen. Existe de hecho un parecido, en tanto la recuperación de información se mide en relación a la fiabilidad de los recursos a los que se acceden, mientras que la comunicación interpersonal se valora en parámetros que van desde el anonimato subiendo en escala según se aumenta el denominado disclosure (lo que mostramos de nosotros mismos en la red). Este paralelismo no se había estudiado en absoluto en el aula. El CMD permitió recoger en el cuestionario final una serie de contestaciones de los estudiantes que habían encontrado dicha correlación. Este aspecto, teniendo en cuenta que el CMD se diseña para mejorar el espíritu crítico del aula, debe considerarse como altamente positivo. Un caso también interesante es la pregunta 7, que cuestiona al estudiante si controla bien su identidad en las redes. Una posible interpretación a la bajada en las respuestas (más dubitativos en el cuestionario final) sería precisamente todo el debate interno que este CMD ha producido en el aula. Sin embargo, no podemos 
descartar una confusión en los conceptos que haya llevado a una mayor parte del grupo a contestar con menor evidencia.

\section{Evaluación del diseño puesto en práctica}

Esta intervención en el aula me ha permitido evaluar aspectos de mi método que creía que eran adecuados para obtener los mejores resultados en el proceso de aprendizaje, pero que, evidentemente, han quedado evidenciados. En primer lugar, la elaboración de un mapa claro de contenidos es imprescindible. A menudo obviamos la triplicidad que el proceso de aprendizaje lleva. Tal es así, que este CMD gira en torno al desarrollo de un contenido actitudinal. Ha sido posible en parte por la naturaleza de la asignatura, que incluye contenidos que se relacionan directamente con el desarrollo personal, como es la imagen que tenemos de nosotros mismos. Es por este motivo que considero este CMD como un avance muy importante en mi docencia, con independencia de que los resultados del pre-post cuestionario no evidencien claramente un avance, lo es si se marcan preguntas claves ( 5 y 7 como se ha dicho). Observo que ciertamente ha habido, en primer lugar, una aceptación de la metodología diseñada, con estudiantes más involucrados mientras avanzaba el CMD. En segundo lugar la participación activa de los estudiantes en los debates y su genuino interés en la reflexión crítica, son los aspectos que más valoro de toda esta experiencia.

Para el resto del curso, la asignatura se seguirá estructurando, muy definida, en torno a los 3 tipos de contenido. Los mismos, ya están publicados en el aula virtual y en el proyecto docente. Otros aspectos del CMD, en concreto el cuestionario inicial y final, me han resultado menos útiles. Y no lo digo tanto por los resultados obtenidos en el contraste, sino por la implementación. Resulta complicado diseñar el cuestionario, aunque se pueda hacer 
sobre la marcha, pues durante el proceso surgen nuevos conocimientos que no esperaba, y que quedan fueran del proceso de evaluación al ser el cuestionario final una repetición del primero. Además, esta técnica implica una inversión importante de tiempo, que resta mi capacidad a otras cuestiones del aula que considero muy importantes.

\section{Referencias bibliográficas}

Ardaiz-Villanueva, O., Nicuesa-Chacón, X., Brene-Artazcoz, O., de Acedo Lizarraga, M. L. S., \& de Acedo Baquedano, M. T. S. (2011). Evaluation of computer tools for idea generation and team formation in project-based learning. Computers \& Education, 56(3), 700-711.

Bain, K. (2006). Lo que hacen los mejores profesores de universidad. Universitat de València.

Finkel, D. (2008). Dar clase con la boca cerrada. Revista electrónica sobre la enseñanza de la Economía Pública Págs, 49, 60.

Hung, W., Jonassen, D. H., \& Liu, R. (2008). Problem-based learning. Handbook of research on educational communications and technology, 3, 485-506.

King, P. M., \& Kitchener, K. S. (1994). Developing Reflective Judgment: Understanding and Promoting Intellectual Growth and Critical Thinking in Adolescents and Adults. Jossey-Bass Higher and Adult Education Series and Jossey-Bass Social and Behavioral Science Series. Jossey-Bass, 350 Sansome Street, San Francisco, CA 94104-1310.

Jornadas de Formación e Innovación Docente del Profesorado | № 1 (2018) Esta obra se distribuye con la licencia Creative Commons 